\title{
Development of a reactive silencer for turbocompressors
}

\author{
N. González Díez, J.P.M. Smeulers, D. Meulendijks \\ TNO \\ Heat Transfer \& Fluid Dynamics \\ The Netherlands
}

\author{
S. König \\ Siemens AG \\ Energy Sector \\ Duisburg/Germany
}

\begin{abstract}
Turbo compressors can generate tonal noise in a frequency range of 1000 to 5000 $\mathrm{Hz}$, which may cause nuisance in the environment where the machine is installed. The cause of this tonal noise is the interaction between the flow and the rotating blades inside the compressor. The noise is transmitted from the compressor into the pipe system at both suction and discharge sides. Then it is radiated from the pipe walls to the environment. As this noise source can't be avoided, if necessary a silencer can be installed between the compressor and the pipe system. The silencer blocks the transmission of the noise. The most common type of silencer for this application is the so-called absorption type. However, due to high flow velocities and vibrations, the absorption material may deteriorate and be blown away. A sturdier silencer type has been developed based on rigid acoustic resonators. In this paper the development process is described. This includes one and three dimensional acoustic modeling, scale model tests and the design for a full scale application. Compared to the absorption type silencer, the final silencer design has similar or lower pressure loss and fits in the same containment.
\end{abstract}

\section{INTRODUCTION}

Large centrifugal compressors applied for gas transport and storage may generate tonal noise in a frequency range where the human ear is most sensitive. Therefore the rules for allowable noise levels are stricter when a dominant tone is present in the noise spectrum radiated by an installation. For instance, the European Commission Environmental Noise Directive [1] requires an additional $5 \mathrm{~dB}$ reduction of the allowable noise level if there is a tonal component in the spectrum. Therefore tonal noise generated by a compressor has to be suppressed or mitigated. One way to do so is by installing a silencer.

The noise pattern generated by a large centrifugal compressor, such as the one shown in Figure 1, is characterized by high frequencies generated by the moving blades of the impeller. Especially when vaned diffusers are applied, the flow disturbances caused by the impeller are converted effectively in acoustic energy at the vanes of the diffuser. Therefore compressors equipped with low-solidity vanes (LSVs) in the diffuser, commonly applied to improve the performance, will show strong tonal noise generation. Motriuk [2] reports an increased noise radiation of compressors with LSV's compared with compressors without vanes in the diffuser.

\footnotetext{
${ }^{1}$ Currently Project Manager at Huisman Equipment BV.
} 
As the compressor itself has very heavy and rigid walls, the noise generated in the compressor will not radiate from the compressor itself, but the acoustic waves will first travel to the discharge and suction pipe system, where they can be radiated from the (relatively) flexible pipe walls. Additionally, vessels and air coolers are objects that easily radiate noise. In order to prevent noise radiation from the connected piping and equipment, a silencer can be placed at the suction and discharge flanges of the compressor. The acoustic waves travelling into the pipe system are thus blocked by the silencer.

The conventional technique for attenuating high frequency noise is by a silencer filled with absorption material - the so-called absorption-type silencer. This is effective for frequencies of $500 \mathrm{~Hz}$ and higher. For lower frequencies, so-called reactive silencers are applied, which are based on resonators like Helmholtz and Standing Wave resonators. The acoustic impedance in the flow path is changed by these resonators, causing the acoustic waves to be blocked. As the frequency of the tonal noise generated by centrifugal compressors is well above $500 \mathrm{~Hz}$, the common solution is to apply an absorption type damper. However, a great disadvantage of this type of silencer is the deterioration of the absorption material. In some cases, this material has even completely disappeared, as is shown for instance in Figure 2. The consequences of this material going into the pipe system need no further explanations. This is also one of the reasons that there is reluctance to install this type of silencer in the suction line of a compressor.

The lack of reliability of the absorption-type silencer has triggered the development of the new type of silencer presented in this paper, which has at least the same performance, but is significantly more robust.

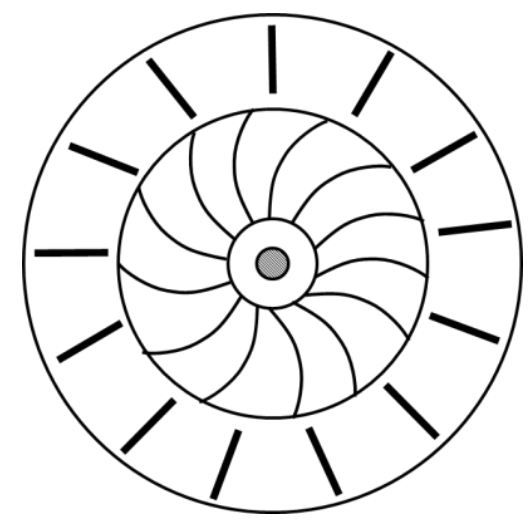

Figure 1 -Schematic of a compressor with diffuser vanes to improve the performance, usually with a penalty on noise radiation. 


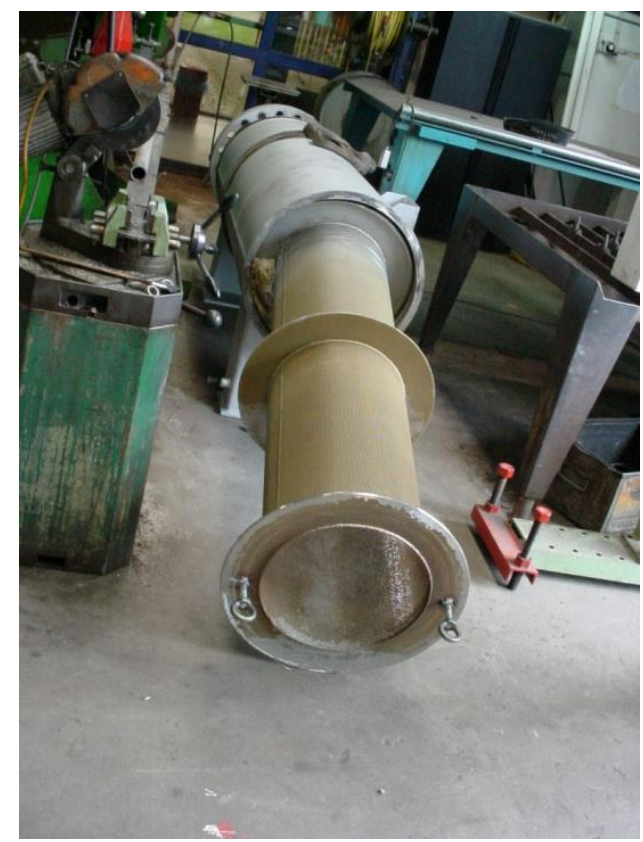

Figure 2 - Absorption-type damper where no absorption material is left.

\section{SILENCING PRINCIPLES}

Essentially there are three principal mechanisms for the reduction of sound in the air or gases in pipes and ducts, either suction or discharge or even fluid filled pipes for that matter. These are absorption, reflection and cancellation. See also Munjal [4].

\subsection{Absorption}

In case of absorption the amplitude of the pressure waves is reduced through energy losses mainly by friction, viscous and thermal effects and sometimes flow in porous material and narrow openings. This can be achieved by materials like mineral wool, steel wool, ceramic sponge, rigid but porous materials, perforated grids or ducts. The effect can be achieved for pass-through situations (gas and sound pass through the material) as well as pass-by situations, i.e. duct linings. Though vibrations in the material itself, in case of non-rigid materials, can add to the desired sound losses, the main effect occurs in the voids within the material. This effectively means that the type of material is less important than the shape and amount of connecting voids [3]. For other properties like temperature resistance the type of material is often of prime importance. In this application, because of the low pressure loss requirement, only the pass-by type silencer comes into consideration.

\subsection{Reflection}

In case of reflection the sound wave is reflected back to the source due to the appropriate (change of) impedance at a given position in the duct. This impedance is achieved by resonant elements, like volumes, side pipes and volume resonators in various combinations. Perfect reflecting conditions can only be achieved at 
specific frequencies. At other frequencies, the effect is either inexistent or even counterproductive (amplification). Furthermore, energy losses in the case of reflection will occur at several positions within the system, caused by friction and flow effects. This will add to the overall noise reduction but is not essential.

\subsection{Cancellation}

In case of cancellation the pressure in the sound wave is - partially - cancelled by another sound wave in a fully opposing phase. In more dimensional cases it is not just a wave but a complete sound field that needs to be cancelled. In some cases this effect can be realized in a passive way by creating the appropriate phase shift between parts of the considered sound wave, but in general this will have to be achieved with an anti-sound source, making just as much sound as the original one. In that case the effect can be constantly adjusted to an optimal performance with varying working conditions.

A silencer can of course use a combination of these three mechanisms. The total performance of such a combination can be more than just the summation of each positive action independently. For instance, adding some absorbing effect at the right spot to reflective silencers can increase their effectiveness; absorption in combination with active noise control can reduce the power requirements for the active system; perforated plates can make absorption more effective.

A schematic view of the pass-through absorption silencer attached to a pipe and its reactive counterpart are shown in Figure 3.

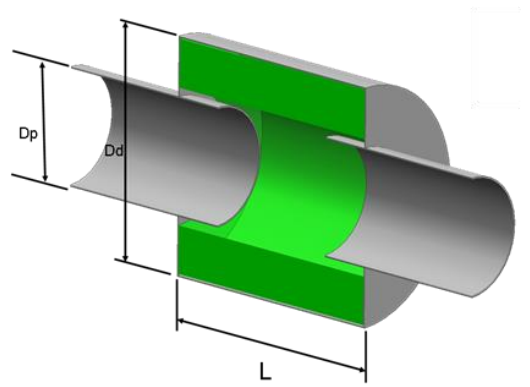

(i) Pass-through absorption silencer

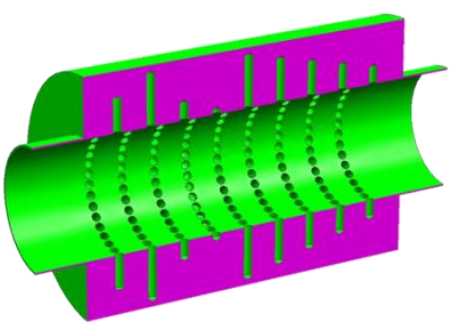

(ii) Resonator-array reflective silencer.

Figure 3 - Schematics of absorption-based and reflection-based silencers.

\section{SILENCER DESIGN}

\subsection{Silencer requirements}

The application envisioned for the eventual design is a centrifugal compressor with Low Solidity Vanes (LSVs) in the diffuser of the last stage. The demands for the silencer are here enumerated:

1. Broadband effectiveness, i.e. a frequency range of 700 to $3000 \mathrm{~Hz}$. This is the frequency range in which the strongest generated frequency component is expected. This is the blade passing frequency (BPF). As the silencer has to be suitable for compressors running at a variable speed, this leads to the wide frequency range.

2. Reduction of tonal noise components below the background noise level, to avoid the $5 \mathrm{~dB}$ penalty applicable for tonal components. 
3. A reduction of the overall sound pressure level of at least $5 \mathrm{~dB}$.

4. Limited pressure loss, which has been specified as a (small) fraction of the gain in head by applying the LSVs. In the ideal case the pressure loss in the silencer is zero.

5. Durable and simple construction. The reliability of the silencer should be high and should require minimal maintenance. In case of polluting gases it should be possible to clean the silencer.

Considering these design requirements and the principles explained in section 2, a choice is made on which silencer type to pursue. Especially the last requirement seems to exclude the application of the traditional acoustic absorption materials, which makes the reactive-type silencer the most sensible choice. The cancellation type is considered to be too complex, because it requires active elements

\subsection{The reflection-type silencer}

The reflection silencer is based on a series of resonators that are located in the wall of a duct, similar to the absorption material in a pass-by silencer (refer to Figure 3 ). The adjacent resonators are tuned to a series of resonance frequencies covering the requested frequency range. The distance between the resonance frequencies depends on the width of the absorption band of each resonator. Each resonator will absorb acoustic energy from the main duct and will dissipate this energy. In fact the resonator will create a near zero pressure point in the main duct. It depends on the size of the resonator to what extent the zero pressure can be achieved and thus the performance of the silencer.

There are two types of resonators that can be applied. These are the Helmholtz $(\mathrm{HH})$ resonator and the standing wave (SW) or quarter wavelength (QWL) resonator. The $\mathrm{HH}$ (Helmholtz) resonator consists of a volume and a pipe. It is the analog of a mass spring system in which the compressibility of the gas in the volume is the spring and the gas in the pipe forms the mass. The QWL (Quarter Wavelength) resonator is based on the standing wave that can build up in a pipe that is open at one side and closed at the other side. The QWL resonator has multiple resonance frequencies, i.e. for $f=(2 n+1) . c / 4 L, n=0,1,2,3, \ldots$ As for this application the frequencies are relatively high (short wavelengths) the QWL resonators are applied for the high frequency range and the $\mathrm{HH}$ resonators for the lower frequencies.

A complication in the design is that for these high frequencies the acoustic waves can propagate in three dimensional modes. This means that the waves not only propagate parallel to the pipe axis but also in transversal direction. In order to improve the effect of the resonators only plane waves are allowed in the silencer. This is achieved by directing the flow through slits with a width smaller than half a wavelength. This concept is shown schematically in Figure 4. In order to keep the pressure loss within acceptable limits the flow velocity in the slits should be low. In the design, the velocity in the slits is the same as in the main pipe. 


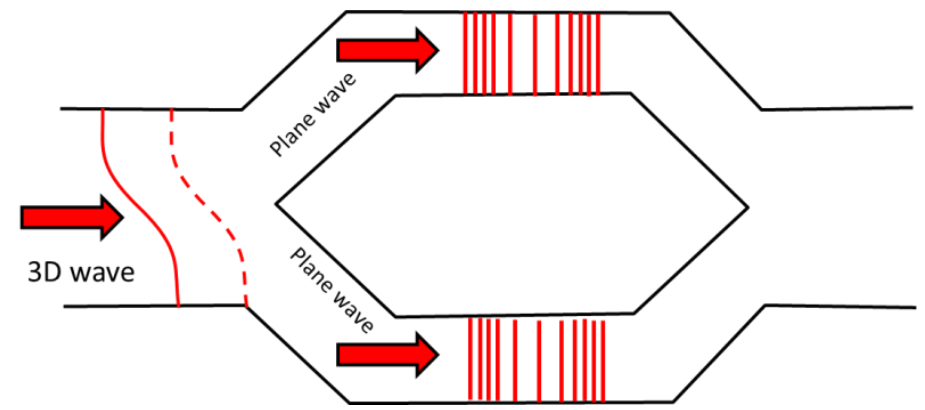

Figure 4 - Flow split concept as a means to remove transversal wave propagation difficulties.
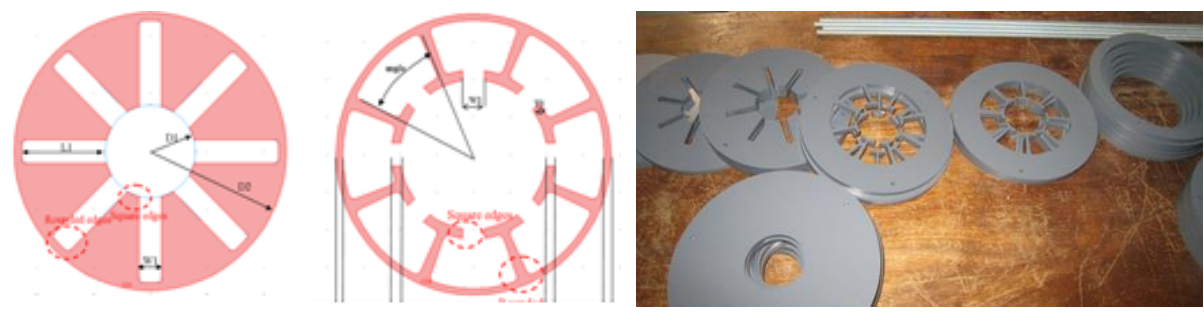

Figure 5 - Scale-model disks with QWL and HH resonators.

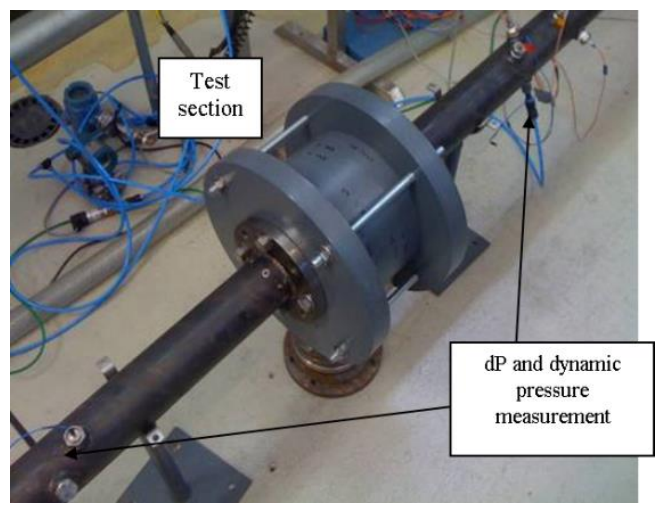

Figure 6 - Test set-up.

\subsection{Stacked disk configuration}

A special construction to configure a resonator array has been invented called the "stacked disk configuration". The configuration consists of a series of plates stacked next to each other. In each plate, a number of cavities is formed that form resonators tuned at specific frequency. A silencer for a specific frequency range can then be created by stacking a series of disks together. The entire assembly is placed in a pressure containment. The assembly itself does not have to sustain 
large pressures. The diameter of the pressure container is relatively small and can be a pipe section with a somewhat larger diameter than the pipe system, which avoids the high costs of the application of a vessel. Examples of disks with resonators (as used in scale model testing explained below) are shown in Figure 5.

\section{ACOUSTIC DESIGN}

The full-scale design of the silencer is based on acoustic modeling with tools of increasing level of detail. In a first step, the silencer is designed following a 1D approach performed with PULSIM [5]. The outcome of this step is a suitable candidate to check with a 3D acoustic solver. The tool used is COMSOL Multiphysics [6]. Additionally, scale-model testing has been done to validate the modeling tools, prove the concept and check performance key indicators such as pressure drop and acoustic transmission loss.

\subsection{Scale-model testing}

Two types of resonator arrays have been tested; one with so-called quarter wavelength resonators (QWL) and one with Helmholtz resonators $(\mathrm{HH})$. The schematics of the design and the test subjects are shown in Figure 5. The stack of disks installed in the test rig is shown in Figure 6.

The test rig consists of an acoustic source, an inlet and outlet pipe terminated with a silencer. At inlet and outlet pipe a Two Microphone Method (TMM) set-up was applied to measure the incoming, reflected and transmitted acoustic waves. Both a 1D and a 3D model was made and the results are compared with the measured Transmission Loss (TL). The results are shown in Figure 7.

For the QWL resonators, both $1 \mathrm{D}$ and 3D models predict the resonance frequencies sufficiently accurate. As expected, the 3D model is more accurate for the higher frequencies. However, because the 1D model includes damping it reproduces the height of the peaks more accurately. In the 3D model the magnitude of the peaks is exaggerated. For frequencies above approximately $2500 \mathrm{~Hz}$ the TMM provides erroneous results due to transversal modes in the inlet and outlet pipe.

For the $\mathrm{HH}$ resonator array the $1 \mathrm{D}$ model overestimates the performance of the silencer, whereas the 3D model is more accurate. Still the minimum performance at approximately $1800 \mathrm{~Hz}$ is satisfactory. This can be improved by bringing in additional $\mathrm{HH}$ resonators tuned at $1600 \mathrm{~Hz}$.

The overall conclusion to this part of the study is that the modelling approach is valid, i.e. the $1 \mathrm{D}$ model can be used to perform fast preliminary design, and the $3 \mathrm{D}$ model can be used to fine-tune or check the design. Attention should be paid to damping though to avoid that the expectation on the performance of the silencer is non-conservative.

\subsection{Full-scale design}

To reach the final goals as set in section 3.1, the design has been optimized using a Pulsim 1D model and subsequently a Comsol model of one segment of the silencer.

The design philosophy is as follows: to minimize the maximum outside diameter, it was decided to use a combination of QWL and $\mathrm{HH}$ for the final design. Especially the lower frequency QWL resonators can become relatively long. Therefore for the lower frequencies $\mathrm{HH}$ resonators are applied and for the higher frequencies QWL. A first design is required with an initial number of centre frequencies. Based on the 
performance of that design, the design is further optimized by shifting the centre frequencies or adding centre frequencies if necessary.

The 1D design, as provided in the previous section is transferred to a 3D Comsol model in order to check the 3D effects on the performance of the design. This model is shown in Figure 8. The QWL for the higher frequencies and the $\mathrm{HH}$ for the lower frequency (downstream of the QWL) can be clearly seen (QWL being narrower and with smaller volumes, $\mathrm{HH}$ downstream with larger volumes).

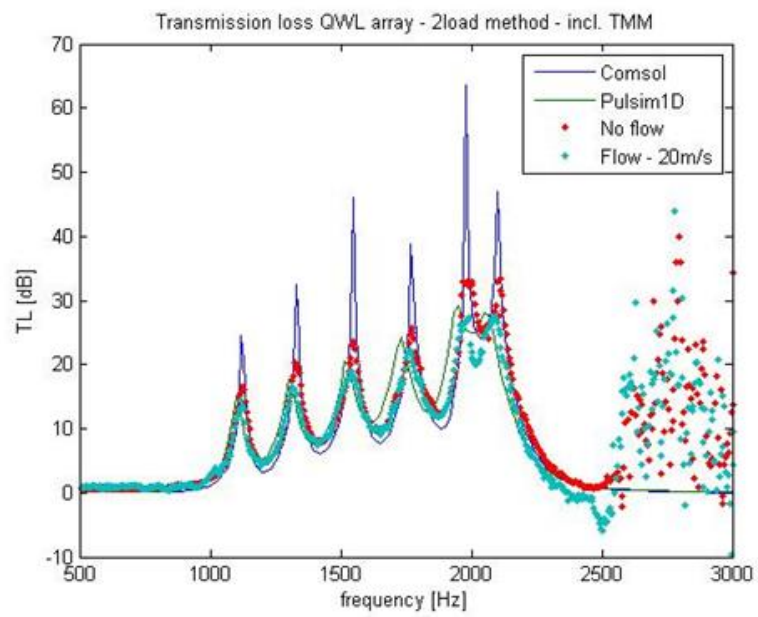

(i) Quarter-Wavelength Resonators

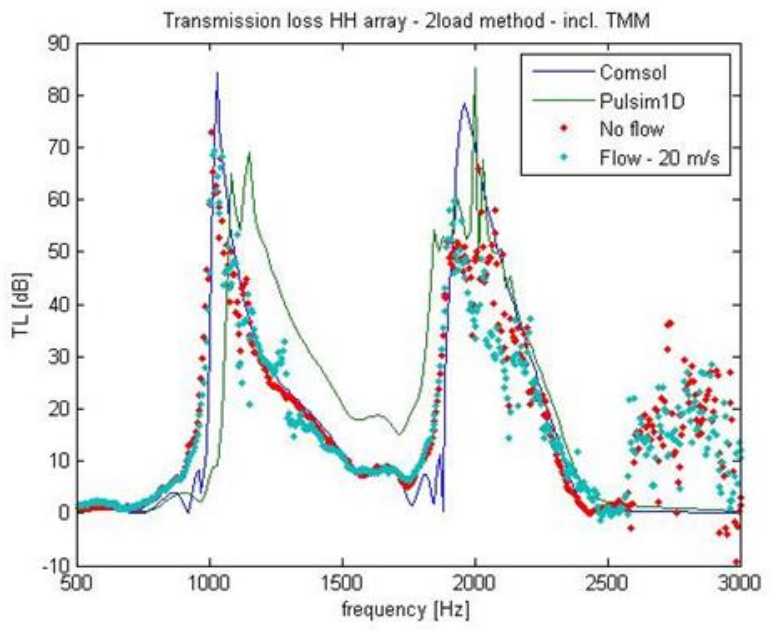

(ii) Helmholtz Resonators

Figure 7 - Comparison of measured and modelled performance of different types of resonator arrays. 
Figure 9 shows the insertion loss for the 1D and 3D simulations of the 1D optimized design and for the 3D simulation of the 3D optimized design. In the final 3D design the IL is larger than $10 \mathrm{~dB}$ for most of the frequencies of interest. This is well above the minimum of $5 \mathrm{~dB}$ required from the design. This margin is expected to be required to cope with the effect of impedance of the compressor stage and the resonances upstream of the silencer. This design is considered satisfactory.

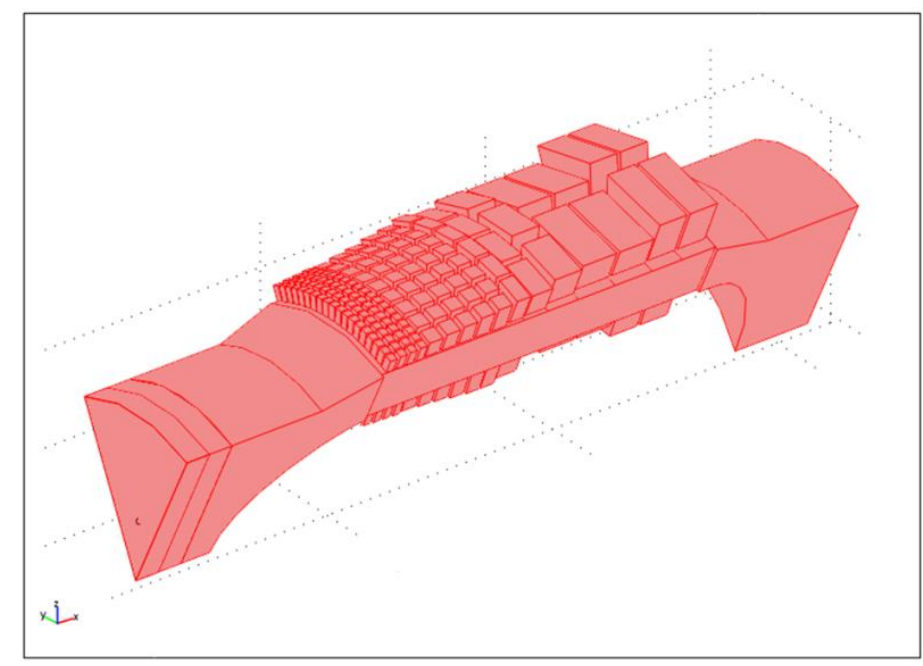

Figure 8 - View of the 3D numerical model with QWL elements upstream the HH elements downstream.

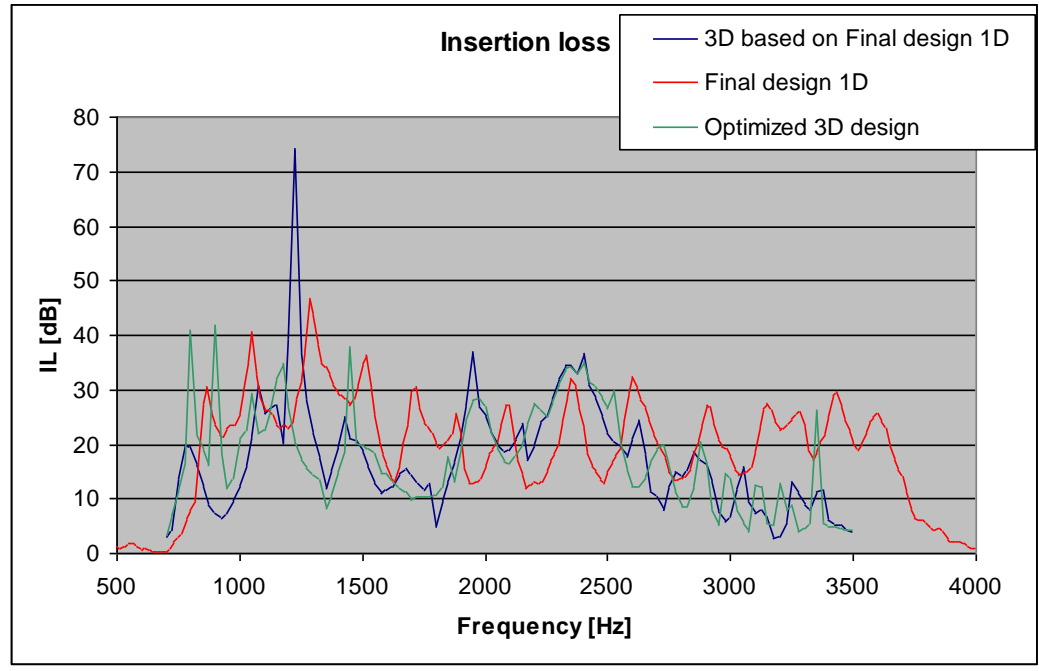

Figure 9 - Comparison of the 1D design, 3D design and further optimized design. 


\section{CONCLUSIONS AND PERSPECTIVES}

The present study has described the acoustic design of a reactive-type silencer. From the results presented above, the following conclusions are extracted:

- A reactive-type silencer is expected to be a good alternative to absorption silencers, with an inherent advantage in terms of reliability and pressure drop.

- Numerical modelling is a plausible approach for acoustic design of reactive silencers. Small-scale tests and simulations show sufficient agreement.

- $\quad 1 D$ design can be used for fast preliminary design, although 3D design is necessary to achieve better accuracy. Capturing damping in such 3D approach is essential to obtain realistic estimates of the performance of the silencer.

- The final optimized design meets the requirements established at the beginning of the design process.

The perspectives in terms of the further development of this technology are:

- The cost of manufacturing is expected to be higher than for traditional silencers. The cost of pressure containment will be approximately the same, but the cost of producing the internals out of solid materials is higher.

- For applications with a limited frequency range, i.e. compressors with a constant speed, the silencer can be considerably smaller than an absorption silencer. The manufacturing costs will be lower in that case.

- Application may also be of interest for screw compressors which show high frequency pulsations with even higher amplitudes.

\section{ACKNOWLEDGEMENTS}

The authors wish to thank Siemens AG Duisburg and TNO for the permission to publish this paper.

\section{REFERENCES}

[1] European Commission: "Directive 2002/49/EC", Netherlands: "Regeling geluid milieubeheer".

[2] Motriuk, R.W., Harvey, D.P., Centrifugal Compressor Modifications and Their Effect on High-Frequency Pipe Wall Vibration, Journal of Pressure Vessel Technology, Vol. $120 \mathrm{nr} .3$, 1998, page 276-282.

[3] Delany, M.A. and Bazley, E.N., "Acoustic Properties of Fibrous Absorbent Materials", Applied Acoustics, vol. 3, 1970, pp. 105-116

[4] Munjal, M.L., Acoustics of Ducts and Mufflers, March 1987, John Wiley \& Sons.

[5] PULSIM see https://www.tno.nl/content.cfm?context=thema\&content= prop_case\&laag $1=895 \&$ laag $2=911$ \&laag3 $=96 \&$ item_id $=1499 \& T a a l=2$

[6] COMSOL Multiphysics, http://www.comsol.com/ 